\title{
Knowledge, awareness, and preventive behaviors toward osteoporosis among a sample of premenopausal women in Erbil city, Iraq
}

\begin{abstract}
Background and objective: Osteoporosis is among the top five conditions causing disability and prolonged hospital stay; however, it is still underestimated worldwide, especially in developing countries. Therefore, this study aimed to determine the knowledge, attitude, and preventive practice in Erbil city toward this global disease.

Methods: A cross-sectional study was conducted among 395 premenopausal women aged $\geq 25$ years and $\leq 49$ years old, who were recruited by simple random sampling throughout three months from the first of October to December 2015. A questionnaire was completed through a direct interview, which consisted of four parts, including questions about knowledge, attitude, practice, and demographic questions. The statistical package for the social sciences was used for data analysis.

Results: The mean age of the participants was $35 \pm 8.24$ years. Almost half of them did not hear about osteoporosis before. The identification of risk factors by participants was poor. Out of 395 of the participants, $92.9 \%$ had no knowledge about the diagnosis of this disease. Approximately half of the participants believed that there is no way to prevent osteoporosis. Regarding practice scoring to prevent the disease, the total score (mean) was 2.9 out of a maximum of 12, which is very low, especially among females aged 25-29 years.
\end{abstract}

Conclusion: The majority of premenopausal women in Erbil city had little knowledge of osteoporosis, however unaware of osteoporosis risk factors and poor preventive practice regarding prevention.

Keywords: Osteoporosis; Knowledge; Premenopause; Disability; Iraq.

\section{Introduction}

Osteoporosis, a serious health problem that affects the quality of life, has been defined as a systemic skeletal disorder in which bone mineral density (BMD) is 2.5 or more standard deviations (SD) below the mean for normal young white women (T-score of -2.5 or less). ${ }^{1,2}$ Currently, osteoporosis is among the top five conditions causing disability and prolonged hospital stay for older people. ${ }^{3}$ Although it is not a curable disease, it is preventable. Hence, increasing knowledge of osteoporosis should be a priority for future intervention programs to promote specific behavioral strategies for osteoporosis prevention, especially it is no longer strict to the advanced age group but has implications for all age groups. In fact, it is a geriatric disease with adolescent onset. ${ }^{4,5}$ Although osteoporosis is more prevalent among Caucasians, it has been estimated that by 2050 , more than 50 percent of all osteoporotic fractures will occur in Asia. ${ }^{6}$ It seems that BMD is lower in most middle eastern countries compared to western countries. ${ }^{7,8}$ National Institute of Health (NIH) suggests that genetic factors may account for up to 75 percent of bone mass, and the remaining 25 percent account for environmental factors. So it is important to understand what is considered a risk factor and who is potentially at a higher risk for bone fragility. ${ }^{9}$ Fractures are

${ }^{1}$ Department of Community Medicine, College of Medicine, Hawler Medical University, Erbil, I raq.

* Correspondence: lara.abbas@hmu.edu.krd 
the hallmark of osteoporosis, and patient education forms an important part of managing this complication. ${ }^{10}$ Without knowledge, these women will not be able to determine their risk of developing osteoporosis, request diagnostic tests, take preventative actions toward warding off this disease, or knowing that osteoporosis starts well before menopause. Therefore, they may think that prevention does not need to take place until after menopause. ${ }^{11,12}$ Therefore, there is an immediate need to increase osteoporosis awareness and subsequent beliefs in older women and younger women. Many interventional studies had shown that enhanced knowledge encourages peoples to seek additional information about osteoporosis and leads to lifestyle changes. $^{13,14}$ This study aimed to find out premenopausal women's knowledge about the symptoms, risk factors, and the complication of osteoporosis. More specifically, it aimed to describe pre menopausal women's attitudes and beliefs toward osteoporosis seriousness and susceptibility of having the disease and determine the knowledge and behaviors of premenopausal women about the preventive measures of osteoporosis.

\section{Methods}

Study design: A cross-sectional study.

Study setting: This study was carried out at four primary health care (PHC) centers in Erbil city, in Iraqi Kurdistan Region; Brayati, Shahedan, Nazdar Bamarni, and Tayrawa PHC centers. These four centers were selected according to their geographical location in Erbil city to cover a large catchment area and make the sample more representative of the Erbil female population.

Study Duration: From September 2015 to April 2016.

Sample size and sampling method: Based on the Daniel (2009) formula ${ }^{15}$ $\mathrm{n}=\mathrm{Z}$ "P(1-P)/d" for calculating sample size and to ensure that the sample is adequate. The expected prevalence of osteoporosis
$(P)$ was set to $0.5(50 \%)$ and for a $95 \%$ confidence interval $(Z 1-\alpha / 2=1.96)$ and precision of 0.05 . This gave a calculated sample size of approximately 384 participants.

Data collection: The researcher attended each PHC center two days per week at the morning shift and sometimes at the afternoon shift. The attended days were not specified, and the research tried to collect an equal sample size in each center (approximately 98 participants at each center). The data were collected at the reception or waiting room of the $\mathrm{PHC}$ centers randomly whenever they were compatible with current study inclusion criteria and wished to participate in the study.

Inclusion criteria: Premenopausal women aged 25-49 years old.

Exclusion criteria: Premenopausal women already diagnosed with osteoporosis and care provider's women.

Data collection tool: The questionnaire had been divided into four sections. The first section included socio-demographic characteristics such as age, menopause, ever married, socio-economic status, education, family history, have ever heard about osteoporosis, and source of information. The second section was bout knowledge (what people know), which included 35 questions about awareness toward osteoporosis, where 20 questions were extracted from the Osteoporosis Knowledge Assessment Tool (OKAT) ${ }^{16}$. The other 15 were modified questions from previous studies. The third section was about attitude (how they feel), which included 15 questions extracted from the Osteoporosis Health Belief Scale (OHBS). ${ }^{17}$ The fourth section was about practice (what they do) and included 14 questions that evaluated osteoporosisrelated life habits adopted from previously utilized studies. The knowledge section was 35 items, each item having "true," "false," and "do not know" options. Each item was coded zero if an incorrect answer or a "do not know" answer was given, and 
one if the correct answer was given, with a total potential score of thirty five. The total score was then divided into three equal categories: knowledge score below (11.7) regarded as low knowledge, (11.7- 23.3) as medium knowledge, and above 23.3 regarded as a high level of knowledge. A five points Likert scale obtained by $\mathrm{OHBS}^{17}$ was used to assess participants' attitude, where score five indicates strong agreement, score one indicates strong disagreement, and zero score was given for do not know the answer. The total score for the 15 questions was 75 . Then total score was divided into three equal categories (25 scores for each): low ( $\leq 25)$, medium (26-50), and high (51-75) level of participants' attitude. Finally, the total potential score of practice was 12, estimated by one point to the most proper answer for each question (question number five and eight were excluded from the scoring). Practice score ranged (1-4) regarded as low practice, (5-8) medium practice, and (9-12) high practice.

Statistical analysis: Data were analyzed using the statistical package for the social sciences (SPSS <Inc., Chicago, III, Version 19). Frequency and percent distribution tables were first presented for all collected variables. Chi-square test of association was used to compare proportions. The Pearson correlation coefficient was calculated to show the strength of correlation between the numerical variables. A $P$ value of $\leq 0.05$ was considered statistically significant.

\section{Results}

A total of 395 premenopausal women participated in the present study.

Participants' socio-demographic characteristic:

The general characteristic of the studied women are shown in Table 1. The mean age was $35 \pm 8.24$. Most of them (329, $83.3 \%)$ were married, and $282(71.4 \%)$ were from urban areas. Only 20 (5.1\%) women were smokers. The results of women's knowledge were assessed by 35 items in questionnaires, summarized in Table 2. This table represents the number of participants who answered the question correctly. So, this study indicates that women are relatively aware of the elderly as the commonest age group affected $(78.7 \%)$, and it leads to an increased chance of bone fracture $(89.4 \%)$. On the other hand, they showed poor knowledge of the other important aspects of osteoporosis, especially questions related to the first symptom of osteoporosis (causing pain $=346$ answered yes) . As also shown in Table 2, there was a discrepancy in a way that their awareness of female gender (59.5\%), early menopause $(51.4 \%)$, and cigarette smoking $(53.4 \%)$ as risk factors of osteoporosis was good. However, they did not have enough awareness toward the race-being white $(46.8 \%)$, family history $(40.8 \%)$, high salt intake $(46.8 \%)$. They also had poor awareness toward the role of corticosteroid $(10.6 \%)$, slimming $(17.2 \%)$, and prolonged amenorrhea (23.3\%). Although most of the participants were aware of advanced age as a risk factor, only $(39.5 \%)$ knew shortening of height in old age as a symptom of osteoporosis. Despite poor recognition of important risk factors of osteoporosis by participants, a high percentage $(73.2 \%)$ scored medium knowledge of osteoporosis in general, while $22 \%$ recorded low score and only $4.8 \%$ had a high knowledge score, as shown in Table 3. As a consequence of this medium knowledge score, it had a significant positive effect on their attitude toward osteoporosis by Pearson correlation analysis as well $(r=0.573$, $P<0.001)$, as shown in Figure 1. 
Table 1: The socio-demographic characteristics of the participants.

\begin{tabular}{|c|c|}
\hline Variables & $N(\%)$ \\
\hline \multicolumn{2}{|l|}{ Age (years) } \\
\hline 25_29 & $137(34.7)$ \\
\hline $30-34$ & $71(18)$ \\
\hline $35-39$ & $47(11.9)$ \\
\hline$\geq 40$ & $140(35.4)$ \\
\hline Mean + Standard Deviation & $35 \pm 8.24$ \\
\hline \multicolumn{2}{|l|}{ Education } \\
\hline Illiterate & $123(31.1)$ \\
\hline Primary & $139(35.2)$ \\
\hline Secondary-Intermediate & $84(21.3)$ \\
\hline Higher education & $49(12.4)$ \\
\hline \multicolumn{2}{|l|}{ Occupation } \\
\hline Employed & $54(13.7)$ \\
\hline Unemployed & $341(86.3)$ \\
\hline \multicolumn{2}{|l|}{ Marital Status } \\
\hline Single & $48(12.2)$ \\
\hline Married & $329(83.3)$ \\
\hline Divorced & $6(1.5)$ \\
\hline Widow & $12(3)$ \\
\hline \multicolumn{2}{|l|}{ Residence } \\
\hline Urban & $282(71.4)$ \\
\hline Rural & $113(28.6)$ \\
\hline \multicolumn{2}{|l|}{ Family History } \\
\hline Yes & $68(17.2)$ \\
\hline No & $327(82.8)$ \\
\hline \multicolumn{2}{|l|}{ Socio economic status } \\
\hline Low & $135(34.2)$ \\
\hline Medium & $201(50.9)$ \\
\hline High & $59(14.9)$ \\
\hline \multicolumn{2}{|l|}{ Smoking } \\
\hline Smoker & $20(5.1)$ \\
\hline Non smoker & $356(90.1)$ \\
\hline Ex-smoker & $19(4.8)$ \\
\hline
\end{tabular}


Table 2: Knowledge of participant about osteoporosis

\begin{tabular}{|c|c|c|c|}
\hline \multicolumn{2}{|c|}{ Question } & \multirow{2}{*}{$\begin{array}{c}\text { Correct } \\
\text { answer }\end{array}$} & \multirow{2}{*}{$\begin{array}{c}\mathrm{N}(\%) \\
\text { Answered } \\
\text { correctly }\end{array}$} \\
\hline 1 & Osteoporosis leads to an increased risk of bone fracture. & & \\
\hline 2 & Osteoporosis usually causes symptoms (e.g., pain) before fractures occur. & False & $49(12.4)$ \\
\hline 3 & $\begin{array}{l}\text { Having a higher peak bone mass at the end of childhood gives no protection against the } \\
\text { development of osteoporosis in later life. }\end{array}$ & False & $340(86.1)$ \\
\hline 4 & Osteoporosis is more common in men. & False & $235(59.5)$ \\
\hline 5 & Cigarette smoking can contribute to osteoporosis. & True & $211(53.4)$ \\
\hline 6 & White women are at the highest risk of fracture as compared to other races. & True & $185(46.8)$ \\
\hline 7 & A fall is just as important as low bone strength in causing fractures. & False & $158(40.0)$ \\
\hline 8 & By age 80 , the majority of women have osteoporosis. & True & $311(78.7)$ \\
\hline 9 & From age 50 , most women can expect at least one fracture before they die. & True & $186(47.1)$ \\
\hline 10 & Any type of physical activity is beneficial for osteoporosis. & False & $127(32.2)$ \\
\hline 11 & It is easy to tell whether I am at risk of osteoporosis by my clinical risk factors. & True & $92(23.3)$ \\
\hline 12 & Family history of osteoporosis strongly predisposes a person to osteoporosis. & True & $161(40.8)$ \\
\hline 13 & An adequate calcium intake can be achieved from two glasses of milk a day. & True & $91(23.0)$ \\
\hline 14 & $\begin{array}{l}\text { Sardines and spinach are good sources of calcium for people who cannot take dairy } \\
\text { products. }\end{array}$ & True & $79(20.0)$ \\
\hline 15 & Calcium supplements alone can prevent bone loss. & False & $66(16.7)$ \\
\hline 16 & Alcohol has an effect on osteoporosis. & True & $238(60.3)$ \\
\hline 17 & A high salt intake is a risk factor for osteoporosis. & True & $185(46.8)$ \\
\hline 18 & There is a small amount of bone loss in the ten years following the onset of menopause. & False & $150(38.0)$ \\
\hline 19 & Hormone therapy prevents further bone loss at any age after menopause. & False & $51(12.9)$ \\
\hline 20 & There are no effective treatments for osteoporosis available in Erbil. & False & $66(16.7)$ \\
\hline 21 & Another name for osteoporosis is osteoarthritis. & False & $173(43.8)$ \\
\hline 22 & Vitamins prevent osteoporosis. & True & $349(88.4)$ \\
\hline 23 & Consumption of cortisone for long duration leads to osteoporosis. & True & $42(10.6)$ \\
\hline 24 & Thin women get osteoporosis more than others. & True & $68(17.2)$ \\
\hline 25 & Sunlight reduces the risk of getting osteoporosis. & True & $358(90.6)$ \\
\hline 26 & Women with osteoporosis shouldn't be physically active. & False & $264(66.8)$ \\
\hline 27 & The blood test should be done for osteoporosis diagnosis. & False & $28(7.1)$ \\
\hline 28 & Women with early menopause are more likely to get osteoporosis. & True & $203(51.4)$ \\
\hline 29 & One of the signs of osteoporosis disease is the shortening of height after the age of 65 . & True & $156(39.5)$ \\
\hline 30 & Milk and yoghurt contain the same amount of calcium. & True & $73(18.5)$ \\
\hline 31 & There is no way to prevent osteoporosis. & False & $192(48.6)$ \\
\hline 32 & Having ovaries surgically removed doesn't affect osteoporosis. & False & $120(30.4)$ \\
\hline 33 & An overactive thyroid doesn't affect the bones. & False & $163(41.3)$ \\
\hline 34 & Prolonged lactation affects bones. & True & $112(28.4)$ \\
\hline 35 & Prolonged amenorrhea not related to menopause affecting Bones. & True & $92(23.3)$ \\
\hline
\end{tabular}


In general, the overall attitude score of the subjects was positive, with a mean score of $44.07 \pm 10.1$. Based on the classification of attitude score, almost three-quarters of the subjects $(70.6 \%)$ had a medium attitude score, as shown in Table 3.

Table 3: Knowledge score, attitude score andpractice score of osteoporosis among 395 participants

\begin{tabular}{lc}
\hline Scores & $\mathbf{N}(\%)$ \\
\hline Knowledge score of osteoporosis (maximum score 35) & $87(22)$ \\
Low <11.7 & $289(73.2)$ \\
Medium 11.7-23.3 & $19(4.8)$ \\
High 23.4+ & \\
Attitude score of osteoporosis (maximum score 75) & $6(1.5)$ \\
Low 1-25 & $279(70.6)$ \\
Medium 26-50 & $110(27.8)$ \\
High 51-75 & \\
Practice score of osteoporosis (maximum score 12) & $324(82)$ \\
Low 1-4 & $68(17.2)$ \\
Medium 5-8 & $3(8)$ \\
High 9-12
\end{tabular}

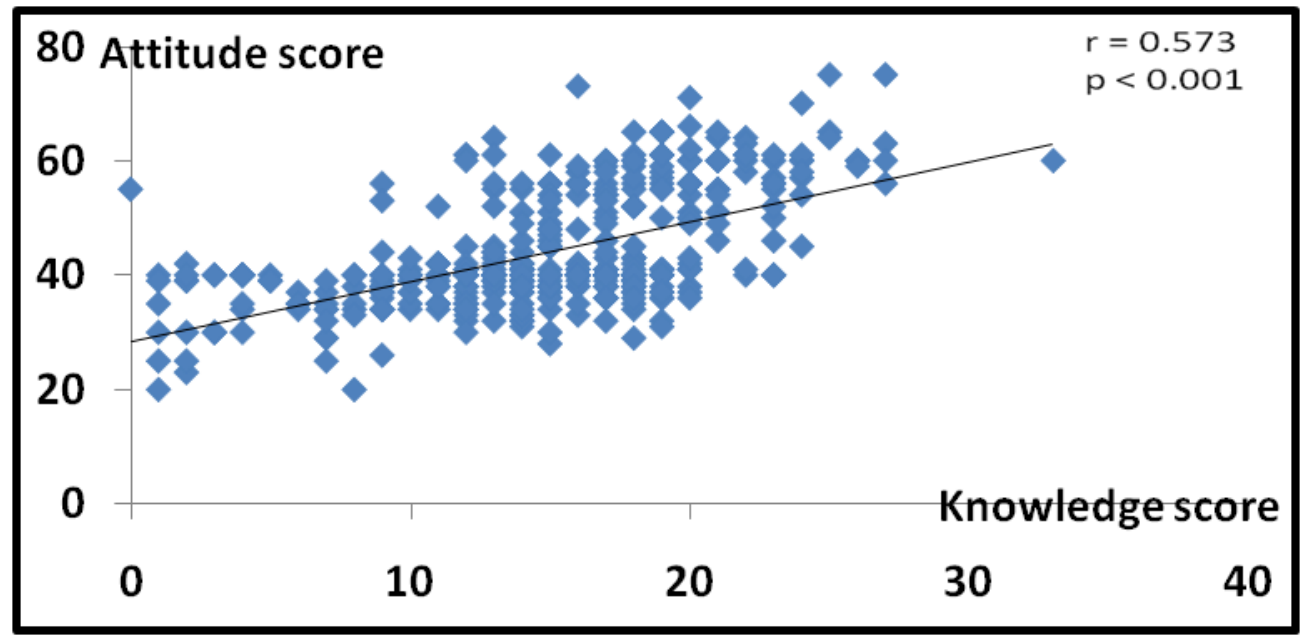

$r=$ correlation coefficient

Figure1: Correlation between knowledge score and attitude score 
Three hundred seven participants did not practice exercise. Among those who practiced exercise, 29 women practiced for less than 15 minutes (Table 4).

\section{Discussion}

Knowledge and belief of participants toward osteoporosis

In general, the study revealed that the majority (two thirds) of women have satisfactory knowledge scores and attitude level scores toward osteoporosis and its prevention. Around $55 \%$ had heard about osteoporosis, similar to the study conducted in Thailand $(50 \%) .{ }^{18}$ However, they failed to follow health promotion activities/practices. This indicates that women's insight toward osteoporosis is not serious, especially when $78.7 \%$ thought that osteoporosis is belonging to the elderly. People who do not perceive themselves at risk for osteoporosis do not practice healthy preventative lifestyle habits. In the present study, awareness of participants toward the role of female gender $(59.5 \%)$, cigarette smoking $(53.4 \%)$, and role of alcohol $(60 \%)$ in osteoporosis were better compared to $\mathrm{Al}$ Shahrani et al. study, ${ }^{19}$ in which the results were $37 \%, 39 \%$, and $23 \%$, respectively. This finding is consistent with previous studies of Al Jalili ${ }^{3}$ and Ungan. ${ }^{20}$ This may own to the general concept of our society toward the bad role of smoking and alcohol consumption on the general condition of the person as a whole, not by knowing its specific role on bone, especially when only $38 \%$ knew about bone loss after menopause and $63 \%$ did not know about the role of hormone replacement therapy in preventing more bone loss. As well as most of them did not indicate about underweight $(17.2 \%)$ as a risk factor for osteoporosis. However, this was the same cognizance of Turkish $(29 \%),{ }^{20}$ American $(27 \%){ }^{21}$ Iranian $(13 \%),{ }^{22}$ and Malaysian $(24 \%)^{23}$ women whom also did not recognize slimming as a risk factor. This also can be attributed to the general concept of people toward obesity as a risk

Table 4: Practice of physical exercise in the studied population.

\begin{tabular}{lc}
\hline Item & $\mathbf{N}(\%)$ \\
\hline Practice exercise & $88(22.3)$ \\
Yes & $307(77.7)$ \\
No & \\
Types of exercise & $8(2)$ \\
Running & $8(2)$ \\
Riding a bicycle & $30(7.6)$ \\
Walking & $38(9.6)$ \\
Swedish (aerobic) exercise & $2(0.5)$ \\
Gardening & $2(0.5)$ \\
Almost all & \\
Frequency of practice exercise/week & $2(0.5)$ \\
Once/week & $34(8.6)$ \\
2-4 times/week & $52(13.2)$ \\
5-7 times/week & \\
Duration of exercise practiced & $29(7.3)$ \\
Less than 15 minutes & $40(10.1)$ \\
$20-30$ minutes & $19(14.8)$ \\
More than 60 minutes & \\
\hline
\end{tabular}


factor for many morbid health problems and always believe that a thin body means better health. In contrast with previous studies investigating osteoporosis, knowledge about osteoporosis amongst women in different countries, including Turkish women's, ${ }^{20}$ American women's, Iranian women's, ${ }^{22}$ and Saudi Arabia women's, ${ }^{19}$ was poor or limited. However, they were expected to have good awareness since most of them had heard of osteoporosis before, even more than our current study. The above results show the necessity of education on the risk factors of osteoporosis because when there is no enough knowledge about risk factors, one does not consider them as serious threats and so does not look for prevention and treatment. Thus, osteoporosis educational program can positively influence participants' belief that they could adopt lifestyle behavioral changes, specifically in calcium intake, smoking cessation, and exercise, regarded as modifiable risk factors.

\section{The preventive practice of participants toward osteoporosis}

Based on our findings, participants knew that regular exercise could protect against osteoporosis, but this knowledge does not translate to practices. Less than one-quarter $(20 \%)$ of the participants were exercising at a minimal level and not regularly. That is why knowledge alone is insufficient to bring about significant improvement in preventive behaviors. Knowledge can influence health related behaviors when mediated by attitudes, beliefs, self-efficacy, and an effective call to action, that is, motivation for positive health. However, various possibilities that explain the weak relationship of knowledge, attitude, and practices have been figured out. These possibilities are the asymptomatic nature of disease until an adverse event occurs, perception of osteoporosis as an irreversible normal aging process, reluctance for bone scanning due to radiation, and perceived health risks. Moreover, various cultural and religious beliefs that can affect an individual's perception of disease and practice of preventive behaviors should also be figured out.

\section{Limitations of the study}

Our sample was restricted to women attending PHC centers, and these women might be more sensitive to their health issues than others in the community. There were interruptions during the time of answering the questionnaire. Most of the questions in the questionnaire were subjective, not objective, that might be misunderstood or regarded as leading questions.

\section{Conclusion}

A considerable number of premenopausal women were unaware of osteoporosis risk factors and its symptoms. However, they were aware of fracture as a complication of it. Most of them regard it as an aging process consequence, and half of them thought there is no prevention. The majority thought there is no effective treatment available in Erbil, ultimately showing a weak practice in prevention, such as inadequate daily calcium intake and low level of physical activity.

\section{Competing interests}

The authors declare no competing interests.

\section{References}

1. Lindsay R, Cosman F, Braunwald E, Facui A, Kasperet D. Osteoporosis, Harrison's Principles of Internal Medicine. Philadelphia: McGraw-Hill; 2001. P. 2226-37.

2. Anthony DW, Bruce P. Burden of major musculoskeletal conditions. Bull World Health Organ 2003; 81:646-56.

3. Jalili Z, Nakhaee N, Askari R, Sharifi V. Knowledge, attitude and preventive practice of women concerning osteoporosis. Iranian J Publ Health 2007; 36(2):19-24.

4. Hossien YE, Tork HM, El-Sabeely AA. Osteoporosis knowledge among female adolescents in Egypt. Am J Nurs 2014; 3(2):1317.

5. Hossien YS. Osteoporosis: knowledge practices and prevention among female adolescent in El-Minia, Egypt. J Res Nurs Midwifery 2014; 3(4):66-72. 
6. Mithal A, Lau E, Dhingra V. The Epidemiology of osteoporosis in Asia. IBMS Bone Key 2009; 6(5):190-3.

7. Maalouf G, Gannagé-Yared MH, Ezzedine J, Larijani B, Badawi S, Rached A, et al. Middle East and North Africa consensus on osteoporosis. J Musculoskelet Neuronal Interact 2007; 7(2):131-43.

8. Kanis JA. On behalf of the World Health Organization Scientific Group: Assessment of osteoporosis at the primary health-care level, Technical report. UK: WHO Collaborating Centre, University of Sheffield; 2007.

9. National Institute of Arthritis \& Musculoskeletal \& skin disease (NIAMS). Osteoporosis: peak bone mass in women; 2009.

10. Harmanjit S, Manoj G, Jasbir S. Osteoporosis: Current Management Guideline. Indian Journal of Clinical Practice 2013; 23(12).

11. Nancy EL. Epidemiology, Etiology \& diagnosis of osteoporosis. Am J Obstet Gynecol 2005; 194 (2):3-11.

12. McLeod KM, Johnson CS. A Systematic Review of Osteoporosis Health Beliefs in Adult Men and Women. Journal of Osteoporosis 2011; 2011.

13. Qi BB, Resnick B, Smeltzer SC, Bausell B. Self-efficacy program to prevent osteoporosis among Chinese immigrants: a randomized controlled trial. Nurs Res 2011; 60:393-404.

14. Chan MF, KO CY. Osteoporosis prevention education programme for women. J Adv Nurs 2006; 54(2):159-70.

15. Daniel WW. Biostatistics: A Foundation for Analysis in the Health Sciences. $8^{\text {th }}$ edition. New York: John Wiley \& Sons; 2009.

16. Winzenberg TM, Oldenburg B, Frendin S, Jones G. The design of a valid and reliable questionnaire to measure osteoporosis knowledge in women: the Osteoporosis Knowledge Assessment Tool (OKAT). BMC Musculoskelet Disord 2003; 4:17.

17. McLeod KM, Johnson CS. A systematic review of osteoporosis health beliefs in adult men and women. Journal of Osteoporosis 2011; 2011:197454.

18. Puttapitakpong $\mathrm{P}$, Chaikittisilpa S, Panyakhamlerd K, Nimnuan C, Jaisamrarn U, Taechakraichana N. Inter-correlation of knowledge, attitude, and osteoporosis preventive behaviors in women around the age of peak bone mass. BMC Womens Health 2014; 14:35.

19. Al Shahrani FM, Al Zahrani AM, Al Haqawi Al. Knowledge of osteoporosis in middle aged and elderly women. Saudi Med J 2010; 31(6):684-7.

20. Ungan M, Tumer M. Turkish women's knowledge of osteoporosis. Fam Pract 2001; 18(2):199-203.

21. Geller SE, Derman R. Knowledge, beliefs, and risk factors for osteoporosis among African-American and Hispanic women. J Natl Med Assoc 2001; 93(1):13-21.
22. Safizadeh $M$, Aminizadeh $E$, Safizadeh $H$. Awareness of osteoporosis among female employees in Kerman, Iran. Russian Open Medical Journal 2015; 4(1).

23. Habibkhan $Y$, Sarriff $A$, Hayatkhan A, Mallhi TH . Knowledge, Attitude and Practice (KAP) Survey of Osteoporosis among Students of a Tertiary Institution in Malaysia. Trop J Pharm Res 2014; 13(1):155

24. National Osteoporosis Foundation. Clinician's Guide to Prevention and Treatment of Osteoporosis. Washington, DC; 2014. 